\title{
Sources and sinks separating domains of left- and right-traveling waves: Experiment versus amplitude equations
}

\author{
Roberto Alvarez, ${ }^{1}$ Martin van Hecke, ${ }^{2, *}$ and Wim van Saarloos ${ }^{2}$ \\ ${ }^{1}$ Department of Physics, Drexel University, 32nd and Chestnut Streets, Philadelphia, Pennsylvania 19104 \\ ${ }^{2}$ Instituut-Lorentz, Leiden University, P.O. Box 9506, 2300 RA Leiden, the Netherlands
}

(Received 18 November 1996)

\begin{abstract}
In many pattern forming systems that exhibit traveling waves, sources and sinks occur that separate patches of oppositely traveling waves. We show that simple qualitative features of their dynamics can be compared to predictions from coupled amplitude equations. In heated wire convection experiments, we find a discrepancy between the observed multiplicity of sources and theoretical predictions. The expression for the observed motion of sinks is incompatible with any amplitude equation description. [S1063-651X(97)51308-0]
\end{abstract}

PACS number(s): 47.54.+r, 03.40.Kf, 47.20.Bp, 47.20.Ky

Since its inception [1], the amplitude equation approach has grown to become an important organizing principle of the theory of nonequilibrium pattern formation-it has not only enabled us to uncover a number of general features of near-threshold pattern dynamics, but it has also allowed us to understand the influence of boundary conditions, defects, etc. Many qualitative and quantitative predictions have been successfully confronted with experiments [2]. The most detailed and successful comparison with experiments has been made for the type of systems for which the theory was originally developed, hydrodynamic systems that bifurcate to a stationary periodic pattern. For traveling wave systems, the range of validity of the appropriate amplitude equation is, however, much more an open question, both because the theoretical derivation has been performed for only a few systems [3], and because direct tests are difficult. Moreover, in practice complications often arise due to the presence of additional important slow variables [4]. Nevertheless, when the amplitude equations include all the necessary terms and respect all the proper symmetries, it is not unreasonable to hope that these equations still provide a good qualitative description outside their proper range of validity; this has indeed been found to be the case in a number of systems (e.g., traveling waves in binary mixtures and liquid crystals $[2,4]$ ).

It is the aim of this paper to point out that sources and sinks that separate patches of traveling wave states can provide a clear way of testing the consistency of the experimental observations with generic qualitative predictions from amplitude equations. Sources and sinks are distinguished by whether the group velocity points outwords or inwards-see Fig. 1. They occur in a wide variety of systems where oppositely traveling waves suppress each other-in directional solidification [5], the printer instability [6], eutectic growth [7], as well as in convection [8,9] — but their properties have remained largely unexplored. We illustrate the idea we put forward with experiments on traveling waves occurring in a liquid heated by a wire just below the surface [8-10]. In the parameter range we have been able to explore, the experi-

\footnotetext{
*Present address: The Niels Bohr Institute and CATS, Blegdams-
} vej 17, 2100 Copenhagen $\varnothing$, Denmark. mental properties of sources and sinks we observe are inconsistent with the behavior predicted by the standard coupled amplitude equations for the near-threshold behavior of onedimensional traveling waves [2],

$$
\begin{aligned}
\left(\partial_{t}+s_{0} \partial_{x}\right) A_{R}= & \varepsilon\left(1+i c_{0}\right) A_{R}+\left(1+i c_{1}\right) \partial_{x}^{2} A_{R} \\
& -\left(1-i c_{3}\right)\left|A_{R}\right|^{2} A_{R}-g_{2}\left(1-i c_{2}\right)\left|A_{L}\right|^{2} A_{R}, \\
\left(\partial_{t}-s_{0} \partial_{x}\right) A_{L}= & \varepsilon\left(1+i c_{0}\right) A_{L}+\left(1+i c_{1}\right) \partial_{x}^{2} A_{L} \\
& -\left(1-i c_{3}\right)\left|A_{L}\right|^{2} A_{L}-g_{2}\left(1-i c_{2}\right)\left|A_{R}\right|^{2} A_{L} .
\end{aligned}
$$

In these equations, we have used suitable units of space and time, and $A_{R}$ is the amplitude of the right traveling mode $e^{-i\left(\omega_{c} t-k_{c} x\right)}$ and $A_{L}$ the one of the left traveling mode $e^{-i\left(\omega_{c} t+k_{c} x\right)}$. Furthermore $\varepsilon$ is the control parameter which measures the distance from the threshold of the instability at $\varepsilon=0$, and the parameters $c_{0}-c_{3}$ are related to the linear $\left(c_{0}\right.$, $\left.c_{1}\right)$ and nonlinear $\left(c_{2}, c_{3}\right)$ dispersion of the waves. It is well known that, strictly speaking, the above equations only arise as the lowest order amplitude equations if the linear group velocity $s_{0}$ is of order $\varepsilon^{1 / 2}$ [11]; nevertheless, the equations are frequently also used when $s_{0}$ is finite at threshold, under the assumption that they still provide an adequate qualitative description [12].

When the coupling parameter $g_{2}$ in Eq. (1) is larger than 1 , the left- and right-traveling waves suppress each other [2], and the system evolves to a state consisting of patches where either $A_{L}$ or $A_{R}$ is zero. Within such a patch, a single amplitude equation suffices, and the group velocity term can be removed by a Galilean transformation. Many experimental and theoretical studies have focused on this situation, in which the theory seems to give a reasonable account of the qualitative observations [2]. Here, we wish to study the sources and sinks that connect these patches of left- and right-traveling waves (see Fig. 1). These coherent structures involve both amplitudes $A_{R}$ and $A_{L}$; therefore the group velocity terms cannot be removed, and they probe the applicability of Eq. (1) to real patterns with finite group velocity $s_{0}$. 


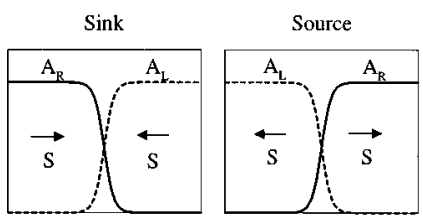

(a)

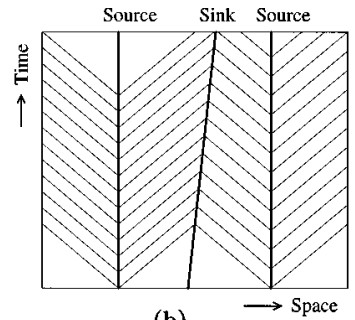

(b)

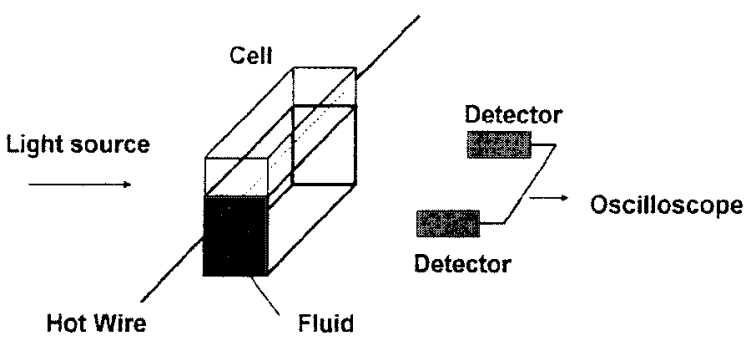

FIG. 1. Definition of sources and sinks. (a) Illustration of sources and sinks as coherent structures in terms of the behavior of the amplitudes $A_{L}$ and $A_{R}$ of the adjacent left- and right-traveling waves. A source is defined as a coherent structure at which waves with total group velocity $s$ pointing outward are generated, and a sink is one between waves with group velocity $s$ pointing inwards. (b) Illustration of the kinematics of sinks and sources in terms of the properties of the adjacent waves, for the case that the group velocity $s$ has the same sign as the phase velocity (as in the experiment, where $s \approx v_{\mathrm{ph}} / 3$ ). In this case, the definition of sources and sinks given under (a) agrees with the intuitive notion that the waves travel away from a source and into a sink. The thin lines indicate lines of constant phase of the traveling waves. In accord with our experimental observations, illustrated in Fig. 3(b), we have drawn a case with two stationary and symmetric sources, each generating waves with different wave numbers and frequencies, and one sink moving according to the phase matching rule, Eq. (2). According to this rule, every constant phase line coming in at the source from the left matches up with a constant phase line coming in from the right.

In our experiments, we have explored the range $0.25 \leqq \varepsilon \leqq 0.5$ - we cannot make accurate measurements below $\varepsilon \approx 0.25$ since the convection then becomes too weak to make careful measurements. Although we therefore cannot study the limit $\varepsilon \rightarrow 0$ systematically, which in principle is necessary to study the validity of (1), we do believe that we effectively probe small amplitude convective patterns: the flow is weak, and there are no systematic trends as $\varepsilon$ is lowered. Moreover, the relative frequency difference of the waves is so small $(1 / 25$ to $1 / 50)$ and the pictures of $[8,9]$ show that in this range sources are wide objects; both observations indicate that an adiabatic approximation appears justified.

Our experimental setup, shown in Fig. 2, is a simple system based on an electrically heated wire immersed below the surface of a fluid [8-10]. Beyond a critical heating power $Q_{c}$, traveling periodic modulations appear at the surface via a forward Hopf bifurcation $[8,9]$. The apparatus is similar to the one used in $[8,9]$ but the design of the Plexiglas cell is somewhat different and larger $(55 \times 15 \times 6 \mathrm{~cm})$, so that the sides are further away from the wire. Both top to bottom and lateral views are possible in our setup; in particular, the lateral view proved especially useful for recording the time series signals. A tungsten wire with a diameter of $0.1 \mathrm{~mm}$ is heated by means of an electrical current and immersed in oil in the middle of the cell and parallel to the longest side. The viscosity of the GE SF 96 silicone oil is $0.5 \quad(1 \mathrm{~S}=1$ $\left.\mathrm{cm}^{2} / \mathrm{S}\right)$. Both the voltage across the wire and the heating current were continuously monitored to check that their values did not change during the measurements. Four springs (two on each end of the cell) provide the necessary tension to keep the wire parallel to the surface of the fluid throughout the cell. A pair of micrometers attached to the ends of the
FIG. 2. Schematic drawing of the experimental setup. The frequencies of the traveling waves can be probed at two positions with photodetectors. The distance between the surface of the liquid and

cell enables us to carefully adjust the wire-surface distance. A shadowgraph technique is used to record the signal generated by the waves. The cell is illuminated with unpolarized white light. Two photodetectors can be placed at adjustable positions along the wire, and the light signal captured by the detectors is sent to a digital oscilloscope. This temporal signal has a very local character as a result of the diverging geometry from the light source towards the acquisition plane. It therefore allows us to measure the local frequency very accurately, even though the relation between the signal and the surface modulations is quite nonlinear due to the strongly inhomogeneous temperature distribution in the direction perpendicular to the wire. Since a single measurement may take several hours due to the long times needed for the fluid to reach a steady state condition, the temperature of the surrounding was carefully controlled.

The picture of the typical sequence of events can be drawn as follows $[8,9]$. Having chosen an adequate depth for the wire, domains of left- and right-traveling waves emerge after the power $Q$ is turned on (our control parameter $\left.\varepsilon \equiv Q / Q_{c}-1\right)$. These patches are separated by sinks and sources. Sources in our experiment send out waves to both sides, while sinks have oppositely traveling waves coming in from both sides. Once transients have died out, the sources stay at some fixed position while the sinks generically move, either towards a source (in which case they usually annihilate each other) or a boundary (thus also disappearing from the scene). A typical example is shown in Fig. 3(b). The time that a simple state, say one with two or three sources and sinks, remains in the cell is quite arbitrary; in the end a source usually is the longest living object [9]. A space-time plot of a source solution in this regime is shown in Fig. 2 of [9], while sideways snapshots of regions of the cell with a source and a sink are shown in Figs. 3(c) and 3(d).

Our main experimental observations concerning the dynamics of sources and sinks are the following: (i) The relative motion of sinks and sources is independent of their separation, and so there does not appear to be a long-range interaction between them. (ii) Sources always have zero velocity, $v_{\text {so }}=0$, and are symmetric: the wave number and frequency of the outcoming left-traveling mode are always the same as those of the outcoming right-traveling mode. The data that illustrate the stationarity of the source are shown in Fig. 3(b), while the fact that the waves emerging from a source are symmetric is illustrated by the photodetector data of Fig. 3(a). In this figure, the frequencies of the signals from the detectors $D_{1}$ and $D_{2}$ at both sides of the left source are the wire was varied from 1 to $3 \mathrm{~mm}$. 


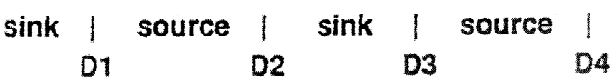

(a)

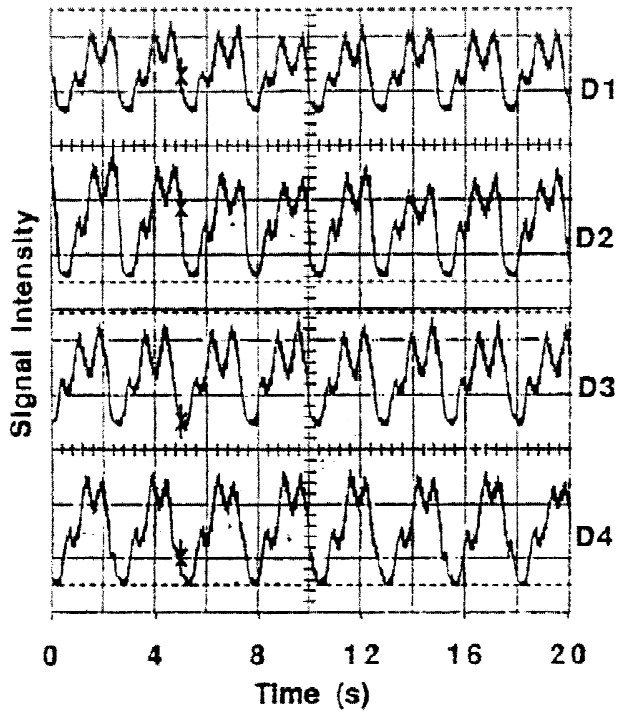

(b)

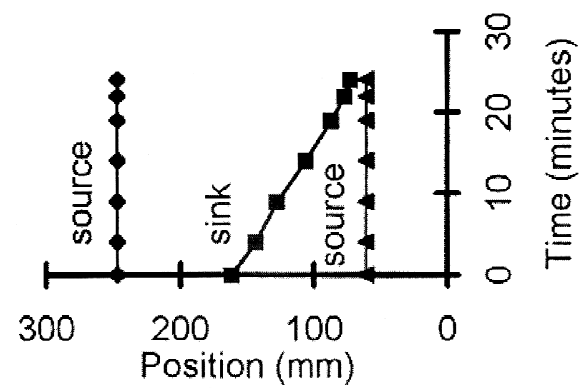

(c)

(d)

FIG. 3. Experimental results. (a) Example of the signals from the photodetectors placed at various positions in between source and sink solutions, as indicated. Typical signal amplitudes are about a factor 8 above the noise level. The absolute value of each signal is arbitrary, especially the frequency is relevant. Note that the frequencies of the two waves sent out by each separate source are exactly the same, but that the two sources send out different waves. As a result, the sink is sandwiched between different incoming waves. (b) Example of traces of the position of sinks and sources in the experiment, as drawn schematically in Fig. 1(b). (c) and (d) Snapshots of regions of the experimental cell with a source (c) and a sink (d), taken from a sideways video image of the cell. Note the symmetry of the pattern around the source, which is roughly in the middle of (c), and the asymmetry of the pattern around the sink, which is just right of center in (d).

exactly the same, and so are those of the $D_{3}$ and $D_{4}$ signals taken around the other source. (iii) While sources are stationary and symmetric, they are not unique: each source sends out waves with a well-defined frequency and wave number, but different sources send out different waves - compare, e.g., the two sources of Fig. 3(a): the frequency of the signals of $D_{1}$ and $D_{2}$ is different from those of $D_{3}$ and $D_{4}$. We take this as evidence that in these experiments at least a oneparameter family of sources exists. (iv) As Fig. 3(b) illustrates, sinks typically move. Moreover, most of our sinks are found to move in such a way that the incoming phases match at the sink $[13,14]$ : in the frame traveling with the sink, the frequencies of the waves coming in from both sides are equal and no phase difference builds up across these sinks. This was already illustrated in Fig. 1(b). If we write the two appropriate incoming modes as $e^{-i\left(\omega_{R} t-i k_{R} x\right)}$ and $e^{-i\left(\omega_{L} t+i k_{L} x\right)}$, then the velocity $v_{s i}^{\text {match }}$ of such a sink is simply

$$
v_{s i}^{\text {match }}=\frac{\omega_{R}-\omega_{L}}{k_{R}+k_{L}}
$$

This relation implies that when a sink is sandwiched between two sources, it moves away from the source with the largest frequency and its velocity is completely determined by the properties of the adjacent sources.

We now confront these results with theoretical predictions. Since the pattern occurs via a forward Hopf bifurcation $[8,9]$, the generic amplitude equations are given by Eqs. (1). We take $g_{2}>1$ since traveling wave states occur, and since the group velocity is about a third of the phase velocity in this experiment, we allow the linear group velocity $s_{0}$ to be of order 1 . Note that for our analysis, we do not need knowledge of the values of the other parameters occurring in Eq. (1).

Property (i), the absence of long-range interactions between sources and sinks, is consistent with the fact that amplitudes at both sides of source and sink solutions approach their asymptotic value exponentially fast, as in the singlemode equation [Eq. (1a)] with $A_{L}=0[15,16]$.

To compare with observations (ii) and (iii), we have analyzed the generic existence and multiplicity of source solutions of Eq. (1) with an extension of previous counting arguments [15] for solutions of the form $A_{R}=e^{-i \omega_{0} t} \hat{A}_{R}(x$ $\left.-v_{\mathrm{so}} t\right)$ and $A_{L}=e^{-i \omega_{0} t} \hat{A}_{L}\left(x+v_{\mathrm{so}} t\right)$. Our analysis [17] shows that independent of the specific values of the parameters, source solutions of Eq. (1) generically come in discrete sets. In particular, one typically expects there to be only a unique symmetric source solution with $v_{\text {so }}=0$, and numerical simulations of Eq. (1) confirm this. This is in clear contradiction with the experiments, where we find a continuous family of them.

We now turn to sinks, which according to (iv) move in the experiments with a velocity (2). Can the phase matching property of the sinks underlying this equation be reproduced at all in an amplitude approach based on Eq. (1)? The answer is no. To see this, note that $v_{s i}^{\text {match }}$ is according to Eq. (2) given in terms of the total frequencies $\omega_{R}, \omega_{L}$ and wave numbers $k_{R}, k_{L}$ of the incoming modes. In an amplitude expansion, these are written as an expansion about their critical values, e.g., $\omega_{R}=\omega_{c}+\omega_{A_{R}}$ where $\omega_{A_{R}}$ is the frequency of the amplitude $A_{R}$ of the right-traveling mode, etc. In terms of these, the experimentally observed velocity of phasematching sinks becomes 


$$
v_{s i}^{\text {match }}=\frac{\omega_{A_{R}}-\omega_{A_{L}}}{2 k_{c}+k_{A_{R}}+k_{A_{L}}},
$$

which underscores once more the fact that this velocity depends on both the fast and the slow spatial scales. However, as an amplitude description is based on an adiabatic elimination of the fast scales, the amplitude equations (1) do not involve the parameters $\omega_{c}$ and $k_{c}$ associated with the fast scales. So, although families of moving sink solutions do exist for Eq. (1), there is no mechanism in these equations to single out the velocity (3) as the selected velocity of sinks.

In summary, our results demonstrate that generic properties of sources and sinks in traveling wave systems, like their multiplicity and dynamics, allow a simple yet powerful comparison between experiments and amplitude equation de- scriptions. For the heated wire convection experiment in the range $0.25 \leqq \varepsilon \lesssim 0.5$ our experiments are inconsistent with an amplitude description. Although a final conclusion must await further study of the $\varepsilon \rightarrow 0$ limit, our results point to two important issues. First of all, they question the soundness of using Eqs. (1) for systems with finite group velocity $s_{0}$. Secondly, they provide a clear example of the possible importance of nonadiabatic effects (coupling of the slow and fast scales [18]) in sinks, even though the two are widely separated, as the relative frequency modulation $\Delta \omega / \omega_{c}$ (which is comparable to the ratio of the typical sink velocity and the phase velocity) can be as small as 1/50 in our experiments.

R.A. is grateful to N. Kwasnjuk for help in constructing the experimental cell.
[1] A. C. Newell and J. A. Whitehead, J. Fluid Mech. 38, 279 (1969).

[2] M. C. Cross and P. C. Hohenberg, Rev. Mod. Phys. 65, 851 (1993).

[3] See, e.g., W. Schöpf and W. Zimmerman, Phys. Rev. E 47, 1739 (1993) for the derivation for binary fluids, and E. Y. Kuo and M. C. Cross, ibid. 47, R2245 (1993) and M. van Hecke and W. van Saarloos, Phys. Rev. E 55, R1259 (1997) for the derivation for rotating convection cells.

[4] For an example in the context of binary mixtures, see $\mathrm{H}$. Riecke, Phys. Rev. Lett. 68, 301 (1992); of chemical waves, see M. Ipsen (unpublished); of convection in liquid crystals, see M. Dennin, M. Treiber, L. Kramer, G. Ahlers, and D. S. Cannell, Phys. Rev. Lett. 76, 319 (1995).

[5] A. J. Simon, J. Bechhoefer, and A. Libchaber, Phys. Rev. Lett. 61, 2574 (1988).

[6] M. Rabaud, S. Michelland, and Y. Couder, Phys. Rev. Lett. 64, 184 (1990); L. Pang and J. R. de Bruyn, ibid. 70, 1791 (1993).

[7] G. Faivre and J. Mergy, Phys. Rev. A 46, 963 (1992).

[8] M. Dubois, F. Daviaud, O. Ronsin, and P. Bergé, Physica D 61, 140 (1992).

[9] J. M. Vince and M. Dubois, Europhys. Lett. 20, 505 (1992).

[10] R. Alvarez (unpublished).
[11] E. Knobloch and J. de Luca, Nonlinearity 3, 575 (1990); C. Martel and J. M. Vega, ibid. 9, 1129 (1996).

[12] H. Riecke, Europhys. Lett. 11, 213 (1990).

[13] Note, however, that Dubois et al. [8] show an example of a weakly oscillating sink close to a source, without matching phases. It is not stated at which value of $\varepsilon$ this occurs, or whether it is a transient phenomenon.

[14] When the phases match across a sink, one might be tempted to say that no phase slips occur there. We prefer to avoid to use the words phase slip in this context, however, as they usually refer to events at which the amplitude goes through zero in a single amplitude equation [15]. In the case of two coupled modes, each separate amplitude does not need to exhibit phase-slip events even if the phases across a sink do not match. The relation between phase slips of the amplitudes and of the patterns is quite different for single and double amplitude equations.

[15] W. van Saarloos and P. C. Hohenberg, Physica D 56, 303 (1992); 9, 209(E) (1993).

[16] T. Bohr, G. Huber, and E. Ott, Europhys. Lett. 33, 589 (1996).

[17] M. van Hecke, C. Storm, and W. van Saarloos (unpublished).

[18] D. Bensimon, B. I. Shraiman, and V. Croquette, Phys. Rev. A 38, 5461 (1988). 